\title{
LA EDUCACIÓN MUSICAL EN LOS ESTUDIOS DEL GRADO EN EDUCACIÓN INFANTIL: ANÁLISIS DE LAS GUÍAS DOCENTES DE LAS UNIVERSIDADES ESPAÑOLAS
}

\author{
THE MUSICAL EDUCATION IN THE EARLY CHILDHOOD EDUCATION \\ DEGREE: ANALYSIS OF THE SPANISH UNIVERSITIES TEACHING GUIDES
}

\author{
José Luis Juárez García \\ joseluis.juarezg@um.es \\ Norberto López Núñez \\ norberto.lopez@um.es \\ Facultad de Educación. Universidad de Murcia (España)
}

Recibido: $15 / 10 / 2020$

Aceptado: 30/06/2021

\begin{abstract}
Resumen:
El presente estudio tiene como objetivo principal analizar las Guías Docentes de las asignaturas del área de Educación Musical en los Grados de Educación Infantil que se imparten en las universidades españolas. Se utilizó un diseño de investigación de tipo descriptivo y exploratorio. La muestra está formada por Guías Docentes pertenecientes a las asignaturas de Educación Musical del Grado de Educación Infantil ( $\mathrm{N}=96)$. Como resultados principales hay que destacar el alto porcentaje de titularidad de universidades públicas respecto a las privadas. También cabe destacar que la mayoría de las asignaturas de Educación musical se imparten en los cursos más avanzados de la titulación. Se concluye que a la hora de elaborar las Guías Docentes no existe un criterio común para ello ya que, aunque la mayoría contienen los mismos apartados, son muy dispares en su contenido.
\end{abstract}

Palabras Clave: Guía Docente; Educación Infantil; Educación Musical; Educación Universitaria.

\begin{abstract}
:
The main objective of this study is to analyze the Teaching Guides of the subjects of the Musical Education area in the Degrees of Early Childhood Education that are taught in Spanish universities. A descriptive and exploratory research design was used. The sample is made up of Teaching Guides belonging to the Music Education subjects of the Infant Education Degree ( $\mathrm{N}=$ 96). As main results, it is necessary to highlight the high percentage of ownership of public universities compared to private ones. It should also be noted that most of the Music Education subjects are taught in the most advanced courses of the degree. It is concluded that when
\end{abstract}


preparing the Teaching Guides there is no common criterion for this since, although most contain the same sections, they are very different in their content.

Keywords: Teacher's guide; Early Childhood Education; Musical Education; University Education.

\section{Introducción}

En la formación del docente de Educación Infantil intervienen múltiples factores, el primero de ellos es la elección de la universidad. La gran cantidad de universidades y la amplia oferta educativa de estudios universitarios genera una competitividad entre la universidad pública y privada en España (Algaba, 2015). Otro de los factores que intervienen según Zabala (2011) son los centros académicos donde se gestan los planes de estudios, horarios, prácticas, apoyo a estudiantes, asistencia y evaluación, todos ellos acordes a un marco común europeo de educación superior. La Comisión Europea implantaba unos procesos de enseñanza centrados en el alumno con unos resultados de aprendizaje formativos añadiendo al objetivo prioritario de mantener el aprendizaje a lo largo de la vida (Wächter, 2004). Como último factor, está el personal docente, encargado de llevar a cabo los planes de estudios mediante sus asignaturas a través de la guía docente. En este sentido, la Declaración de Bolonia entiende la Guía Docente como una herramienta básica del Sistema Europeo de Transferencia de Créditos (ECTS) para alcanzar el objetivo de la cooperación europea a través del desarrollo de metodologías comparables entre estados miembros. En la misma línea, Sánchez-Bascones, Ruíz-Esteban y Pascual-Gómez (2011) definen la Guía docente como una planificación detallada de una asignatura que ordena el desarrollo de una materia o módulo, proporcionando una selección ordenada de contenidos académicos y un planteamiento didáctico de los mismos.

Para contextualizar el presente estudio, se destaca que son muy escasas las aportaciones científicas relacionadas con el estudio de las guías docentes en el ámbito de la educación superior universitaria. La mayoría de los estudios relacionados con las Guías Docentes de las asignaturas universitarias consisten en la creación de instrumentos de evaluación de las mismas, y existen menos investigaciones sobre su creación. Vejo (2012) defendió en su Tesis Doctoral titulada Herramientas para la evaluación, a través de indicadores, de las guías docentes universitarias: aplicación a los estudios de máster de ingeniería ambiental, el diseño y adecuación de una herramienta para la evaluación de los programas universitarios. Para ello, creó un instrumento que permite a los docentes evaluar, de forma simple, las Guías Docentes de sus asignaturas. Por otro lado, Pastor (2009), nos habla de la adecuación del diseño curricular a las exigencias del nuevo marco de la Educación Superior Europea. García y Bernabé (2019) realizan investigaciones acerca de la formación y preparación musical en el Grado de Educación Infantil, cuyos objetivos eran "conocer la opinión de las estudiantes del Grado de Educación Infantil sobre la importancia del hecho musical en el aula, y reflexionar sobre la auto-percepción de su preparación para desarrollar el currículum a través de procedimientos y recursos musicales" (p. 131).

Partimos de la idea que la Educación Infantil es una etapa educativa compleja, dinámica y local, compuesta por diferentes agentes (familia y escuela entre ellos) que tienen relación e influencia entre sí, y tienen como objetivo la mejora del desarrollo y de las competencias de los niños de cero a seis años (García et al, 2001). Se entiende por Educación Infantil, una etapa educativa con identidad propia que atiende a niñas y niños desde el nacimiento hasta el paso a la escuela Primaria. Esta etapa se ordena en dos ciclos. El primero comprende hasta los tres años, y el segundo, desde los tres a los seis años de edad. Dicha etapa es de carácter voluntario y el segundo ciclo de la misma, es de carácter gratuito según el Real Decreto 1630/2006. El mismo decreto, en su Artículo 2 define que la finalidad de la Educación Infantil es contribuir al desarrollo físico, afectivo, social e intelectual de los niños y las niñas. En ambos ciclos se atenderá 
progresivamente al desarrollo afectivo, al movimiento y los hábitos de control corporal, a las manifestaciones de la comunicación y del lenguaje, a las pautas elementales de convivencia y relación social, así como al descubrimiento de las características físicas y sociales del medio. Además, se facilitará que niñas y niños elaboren una imagen de sí mismos positiva y equilibrada y adquieran autonomía personal. La música forma un papel fundamental en ese proceso de formación de un niño de forma integral (Lizaso, 2017). Dada su importancia, resulta imprescindible su inclusión en el currículo de Educación Infantil, que actualmente en España está regulado por el Real Decreto 1630/2006, de 29 de diciembre, por el que se establecen las enseñanzas mínimas del segundo ciclo de Educación infantil.

En el análisis del Real Decreto 1630/2006, encontramos en su Artículo 5. Contenidos Educativos y currículo, que corresponde a las Administraciones Educativas fomentar una primera aproximación a la expresión musical. Pero es en su Anexo, donde más profundiza en las distintas áreas de Educación Infantil. Concretamente nos centramos en el área Lenguajes: comunicación y representación, que es donde se desarrolla la Educación Musical, ya que se tienen en cuenta los diversos tipos de lenguajes, como es el musical. Además, el Real Decreto 1630/2006, cita textualmente:

El lenguaje musical posibilita el desarrollo de capacidades vinculadas con la percepción, el canto, la utilización de objetos sonoros e instrumentos, el movimiento corporal y la creación que surgen de la escucha atenta, la exploración, la manipulación y el juego con los sonidos y la música. Se pretende estimular la adquisición de nuevas habilidades y destrezas que permitan la producción, uso y comprensión de sonidos de distintas características con un sentido expresivo y comunicativo, y favorezcan un despertar de la sensibilidad estética frente a manifestaciones musicales de distintas características (p.13).

Dentro de los bloques de contenidos del área Lenguajes: comunicación y representación, en su Bloque 3. Lenguaje artístico, cita como uno de sus contenidos (p.15):

Exploración de las posibilidades sonoras de la voz, del propio cuerpo, de objetos cotidianos y de instrumentos musicales. Utilización de los sonidos hallados para la interpretación y la creación musical.

Reconocimiento de sonidos del entorno natural y social, y discriminación de sus rasgos distintivos y de algunos contrastes básicos (largo-corto, fuerte-suave, agudo-grave).

Audición atenta de obras musicales presentes en el entorno. Participación activa y disfrute en la interpretación de canciones, juegos musicales y danzas.

Uno de los criterios de evaluación del Real Decreto 1630/2006 es Expresarse y comunicarse utilizando medios, materiales y técnicas propios de los diferentes lenguajes artísticos y audiovisuales, mostrando interés por explorar sus posibilidades, por disfrutar con sus producciones y por compartir con los demás las experiencias estéticas y comunicativas. Con este criterio se evalúa el desarrollo de las habilidades expresivas por medio de diferentes materiales, instrumentos y técnicas propios de los lenguajes musical, audiovisual, plástico y corporal (p.16).

En cuanto a la inclusión de elementos en las Guías Docentes de Educación Infantil son numerosos los autores que han considerado la música como área esencial para el fomento de diversas competencias. Por ejemplo, Alsina y De la Creu (2009) han realizado estudios acerca del desarrollo de la competencia creativa. A esta competencia, Carrillo (2015) suma las competencias relacionadas con la interpretación, la dirección instrumental y el análisis musical. Para incluirlas en las aulas de Educación Infantil es básico que el profesorado esté correctamente formado. Por ello, la inclusión de estas competencias dentro de las guías de las asignaturas de música del Grado de Educación Infantil resulta primordial. En este sentido, la Orden 
$\mathrm{ECl} / 3854 / 2007$ estable como competencia clave relacionada con lo didáctico y disciplinar en materia de música una sola competencia para ser incluida en las guías docentes:

Conocer los fundamentos musicales, plásticos y de expresión corporal del currículo de esta etapa así como las teorías sobre la adquisición y desarrollo de los aprendizajes correspondientes. Conocer y utilizar canciones para promover la educación auditiva, rítmica y vocal. Saber utilizar el juego como recurso didáctico, así como diseñar actividades de aprendizaje basadas en principios lúdicos. Elaborar propuestas didácticas que fomenten la percepción y expresión musicales, las habilidades motrices, el dibujo y la creatividad. Analizar los lenguajes audiovisuales y sus implicaciones educativas. Promover la sensibilidad relativa a la expresión plástica y a la creación artística ( $p$. 53738).

En esta línea, López (2008) y Carbajo (2009) coinciden en recomendar la interpretación y el trabajo sobre la voz como Competencias imprescindibles musicales en el aula de Educación Infantil. Por ello, este elemento también debe ser incluido en las Guías Docentes de Educación Musical.

Por tanto, es necesario que el profesorado adquiera las competencias relacionadas con la Educación musical para que pueda tomar conciencia de la variedad de posibilidades que ofrece la música. Así se disminuiría la baja comprensión que existe sobre la naturaleza musical y las carencias en formación de procedimientos y habilidades que tiene el profesorado al respecto (López, 2008). Un estudio realizado por Vicente y Rodríguez (2014) demostró cómo el profesorado de Educación Infantil no se encuentra preparado para utilizar la música en sus clases ya que dicho profesorado considera que su formación en la universidad fue muy pobre e insuficiente. En cuanto a la formación continua que se les ofrece, también consideran que es escasa. En esta línea, Capistrán (2016) insiste en la falta de formación artística del futuro profesorado. Además, señala la importancia de la música como eje curricular para la formación del futuro docente de Infantil.

\section{Método}

\subsection{Objetivos}

El objetivo principal es analizar las Guías Docentes de las asignaturas destinadas a la formación musical en los Grados de Educación Infantil que se imparten en las diferentes universidades públicas y privadas de España.

Como objetivos específicos tenemos:

1. Señalar los aspectos generales de las Guías Docentes de las materias destinadas a la formación musical en los Grados de Educación Infantil.

2. Identificar los distintos aspectos curriculares que componen las Guías Docentes de las materias destinadas a la formación musical en los Grados de Educación Infantil.

3. Comparar las Guías Docentes de las materias destinadas a la formación musical en el Grado de Educación Infantil entre la universidad pública y privada.

\subsection{Muestra}

La muestra objeto de estudio se compone del total de las Guías Docentes de las asignaturas del área de Educación Musical en los Grados de Educación Infantil que se imparten en las diferentes universidades públicas y privadas de España. El Grado de Educación Infantil se imparte en un 
total de 108 universidades ${ }^{1}$, de las cuales 77 son públicas, y 31 son privadas. Como algunas universidades tienen varios campus y sus guías docentes son iguales, la muestra quedó reducida a 96 guías docentes ( $\mathrm{N}=96$ ), lo que significa un $88,88 \%$ del total de guías de las asignaturas de Música que se imparten en los Grados de Educación Infantil en España. De las cuales 71 eran de carácter público $(73,96 \%)$ y 25 de carácter privado $(26,06 \%)$.El tipo de muestreo realizado fue no probabilístico e intencional o de conveniencia, ya que se utilizaron las Guías Docentes que se consideraron oportunas para su estudio, por lo que es una muestra muy específica (Alaminos, 2006).

\subsection{Instrumento}

Para analizar las Guías Docentes se diseñó un Protocolo de análisis de datos ad hoc en forma de cuestionario digital que pretendía recoger tanto aspectos generales de las asignaturas, como información de los distintos elementos curriculares que componen las Guías Docentes de las mismas ${ }^{2}$.Teniendo en cuenta estas aportaciones se diseñó un protocolo formado por 19 ítems, los cuales estaban divididos en dos bloques diferenciados: uno que aludía a aspectos generales que nos ayudan a identificar una asignatura y otro de los diferentes elementos curriculares que nos aportan las asignaturas. Para llevar a cabo la validación del protocolo de análisis de datos se realizó un proceso de validación por Comité de Expertos, siendo la forma más apropiada para este tipo de protocolos como afirman Millan y Schumacher (2005). El comité ha estado formado por siete profesores, todos ellos doctores, de reconocido prestigio en el ámbito de la didáctica de la educación musical en la etapa de educación superior universitaria y pertenecientes a diferentes universidades españolas. El panel de expertos valoró mediante una escala Likert de 5 puntos el grado de adecuación (4,1 sobre 5$)$ y pertinencia $(4,42$ sobre 5$)$ de los indicadores elaborados que conforman las variables. Se obtuvo un valor muy alto y se calculó el grado de fiabilidad mediante el Alfa de Cronbach obteniendo .783 sobre 1, lo que demuestra que las variables de nuestro instrumento de recogida de datos tienen una buena fiabilidad siguiendo el criterio de autores como Celina y Campo-Arias (2005) y Bojórquez, López, Hernández y Jiménez (2013).

\section{Resultados}

\subsection{Aspectos generales}

En relación con la provincia donde se imparten las asignaturas, están repartidas por todo el territorio español, en las distintas universidades tanto públicas, como privadas. Podemos destacar a la Universidad Pública de Alicante con 5 asignaturas del área de Educación Musical, al igual que la Universidad de Girona, la Universidad de Madrid y la Universidad de Salamanca con 4 asignaturas respectivamente. En cuanto a las Universidades privadas, la Universidad Ramón Llul destaca por encima del resto con un total de 4 asignaturas.

Los nombres que reciben las asignaturas son diferentes, para las 96 Guías Docentes, encontramos 74 títulos distintos. Podemos destacar que el $52.08 \%$ de las asignaturas llevan incorporada la palabra "Música" en su nombre, el 39.5\% la palabra "Musical" y el $8.42 \%$ tienen un nombre diferente. El nombre de la asignatura que más se repite es el de Educación musical, lo que equivale a un $6.3 \%$ del total, puesto que se da en 6 asignaturas.

En cuanto a la titularidad de la universidad, el $76 \%$ son de carácter público (71) y el $24 \%$ son de carácter privado (25).

\footnotetext{
${ }^{1}$ Qué estudiar y donde en la universidad:

https://www.educacion.gob.es/notasdecorte/busquedaSimple.action

${ }^{2}$ https://encuestas.um.es/encuestas/Mjg3ODM.w
} 
Como se puede apreciar en la Tabla 1, las asignaturas están repartidas a lo largo de los cuatro años en los que se imparte el Grado. El curso con mayor presencia de asignaturas de música es 3o con un $41,17 \%$, seguido de 4 o con un $29,2 \%$, segundo y primero son los cursos con menor presencia de asignaturas relacionadas con la música con un $17,7 \%$ y $11,5 \%$ respectivamente. Podemos ver cómo tanto en la universidad pública como en la privada lo más frecuente es a incluir las asignaturas de música en los cursos más avanzados del Grado. En la universidad pública el $42.3 \%$ de las asignaturas se da en 3 으 curso, mientras que $11.3 \%$ se imparte en 1 으. En cuanto a la universidad privada, el $40.0 \%$ de las asignaturas se da en 30 , mientras que $12.0 \%$ se imparte en 1ㅇ. Tanto la universidad pública como la privada siguen la misma tendencia.

Tabla 1

Curso en el que se imparte la asignatura

\begin{tabular}{ccccccc}
\hline Cursos & \multicolumn{2}{c}{ Todas las universidades } & \multicolumn{2}{c}{ Universidad Pública } & \multicolumn{2}{c}{ Universidad Privada } \\
\hline & Frecuencia & Porcentaje & Frecuencia & Porcentaje & Frecuencia & Porcentaje \\
\hline Primero & 11 & 11.5 & 8 & 11.3 & 3 & 12.0 \\
Segundo & 17 & 17.7 & 11 & 15.5 & 6 & 24.0 \\
Tercero & 40 & 41.7 & 30 & 42.3 & 10 & 40.0 \\
Cuarto & 28 & 29.2 & 22 & 31.0 & 25 & 24.0 \\
Total & 96 & 100,0 & 71 & 100.0 & & 100.0 \\
\hline
\end{tabular}

En cuanto a la tipología de las asignaturas, el 66,7\% de las asignaturas son de carácter obligatorio, mientras que el 33,3\% de las asignaturas son de carácter optativo (Figura 1).

Cabe destacar las diferencias de proporción entre obligatorias y optativas entre la universidad pública y la universidad privada. En la pública el $70.84 \%$ de las asignaturas son de carácter obligatorio mientras que el $29.16 \%$ restante son de carácter optativo. En la privada el $84 \%$ de las asignaturas son de carácter obligatorio mientras que el $16 \%$ son de carácter optativo.

- Obligatoria $\quad$ Optativa

64

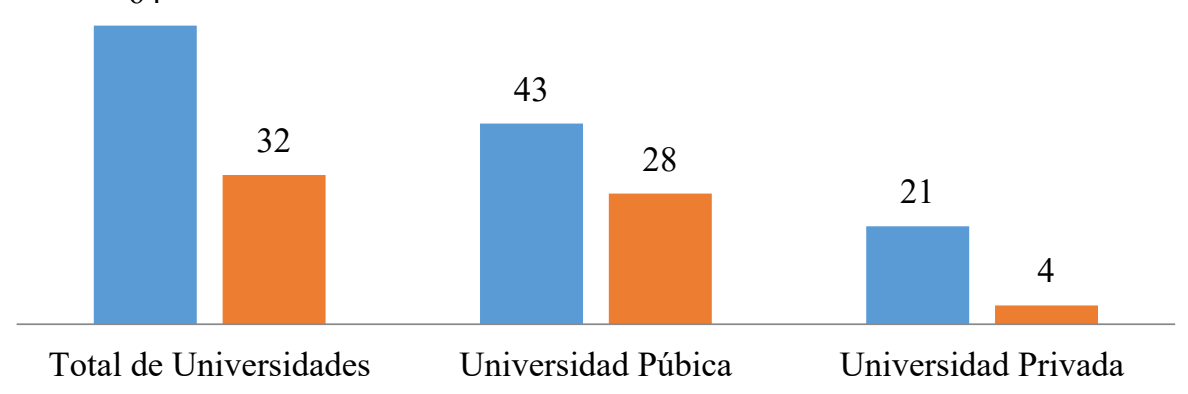

Figura 1. Tipología de las asignaturas.

Con relación a la temporalidad de las asignaturas, como se puede apreciar en la Tabla 2, vemos que el 93,8\% de las asignaturas son cuatrimestrales/semestrales mientras que un 6,3\% de las asignaturas son de carácter anual. Por otro lado, de las asignaturas que son cuatrimestrales/semestrales, el $43 \%$ se imparten en el primer semestre y el $30,2 \%$ de las asignaturas se imparten en el segundo semestre. El resto de las guías no especifica si se imparten en el primer o segundo semestre. Cabe destacar que en la universidad privada el $100 \%$ de las asignaturas son de carácter semestral y que el $44 \%$ se imparten en el según cuatrimestre. En cambio, en la universidad privada, aunque un $91.5 \%$ de las asignaturas son semestrales, el $8.5 \%$ son de carácter anual. 
Tabla 2

Temporalidad de las asignaturas

\begin{tabular}{ccccccc}
\hline Temporalidad & \multicolumn{2}{c}{ Todas las universidades } & \multicolumn{2}{c}{ Universidad Pública } & \multicolumn{2}{c}{ Universidad Privada } \\
\hline & Frecuencia & Porcentaje & Frecuencia & Porcentaje & Frecuencia & Porcentaje \\
\hline Semestral & 90 & 93.8 & 65 & 91.5 & 25 & 100.0 \\
Primer & 42 & 43.8 & 34 & 47.9 & 8 & 32.0 \\
Semestre & & & & & & \\
Segundo & 29 & 30.2 & 18 & 25.4 & 11 & 44.0 \\
Semestre & & 6.3 & 6 & 8.5 & 0 & 0.0 \\
Anual & 6 & 100.0 & 71 & 100.0 & 25 & 100.0 \\
Total & 96 & & & & & \\
\hline
\end{tabular}

En cuanto al número de créditos ECTS de cada asignatura, como podemos apreciar en la Tabla 3 , el $83,3 \%$ de las asignaturas tienen 6 créditos ECTS mientras que el 16,7\% de las asignaturas restantes tienen otras cantidades muy inferiores respecto al número de créditos. En la universidad privada el $92 \%$ de las asignaturas tienen 6 créditos, y tan solo el $8 \%$ tienen menos. En cambio, en la universidad pública, aunque el $80.3 \%$ tienen 6 créditos, hay dos asignaturas que tienen un número elevado de créditos, siendo 9 y 15 respectivamente.

Tabla 3

Número de créditos ECTS de la asignatura

\begin{tabular}{ccccccc}
\hline № Créditos & \multicolumn{2}{c}{ Todas las universidades } & \multicolumn{2}{c}{ Universidad Pública } & \multicolumn{2}{c}{ Universidad Privada } \\
\hline 3 & Frecuencia & Porcentaje & Frecuencia & Porcentaje & Frecuencia & Porcentaje \\
4 & 4 & 4.2 & 3 & 4.2 & 1 & 4.0 \\
5 & 3 & 3.1 & 2 & 2.8 & 1 & 4.0 \\
6 & 4 & 4.1 & 4 & 5.6 & - & - \\
8 & 80 & 83.3 & 57 & 80.3 & 23 & -92.0 \\
9 & 3 & 3.1 & 3 & 1.4 & - & - \\
15 & 1 & 1 & 1 & 1.4 & - & - \\
Total & 1 & 1 & 1 & 100.0 & 25 & 100.0 \\
\hline
\end{tabular}

Con relación al idioma en el que se imparten las asignaturas podemos apreciar en la Tabla 4 como el $72,9 \%$ de las asignaturas se imparten en español, el $17,7 \%$ en catalán, el $6.3 \%$ en otras lenguas cooficiales del Estado como el euskera y el gallego. El 3,1\% restante son asignaturas bilingües y se imparten en español-inglés. También podemos apreciar cómo solo en las universidades privadas imparten algunas asignaturas de forma bilingüe y que a su vez estas no imparten ninguna asignatura en idiomas como el euskera o el gallego. Solo imparten el $20 \%$ en catalán. 
Tabla 4

Idioma en el que se imparten las asignaturas

\begin{tabular}{ccccccc}
\hline & & & & & \\
\hline Español & 70 & 72.9 & 53 & 74.6 & 17 & 68.0 \\
Catalán & 17 & 17.7 & 12 & 16.9 & 5 & 20.0 \\
Euskera & 2 & 2.1 & 2 & 2.8 & - & - \\
Gallego & 4 & 4.2 & 4 & 5.6 & - & - \\
Español- & 3 & 3.1 & - & - & 3 & 12.0 \\
Inglés & & 100,0 & 71 & 100.0 & 25 & 100.0 \\
Total & 96 & &
\end{tabular}

En referencia a las recomendaciones que tienen las Guías Docentes de las asignaturas, podemos observar en la Figura 2 como el $81 \%$ de las Guías Docentes no tienen ninguna recomendación, en el $11,5 \%$ existe la necesidad de tener conocimientos previos y en el $7 \%$ restante existe la necesidad de haber cursado alguna asignatura previamente. No existen apenas diferencias entre la universidad pública y la privada, ya que los porcentajes de ambas son similares. El $80.3 \%$ de las asignaturas de la universidad pública no tienen ninguna recomendación previa frente a un $84 \%$ de la universidad privada. El $12.7 \%$ de las asignaturas de la universidad pública requiere la necesidad de conocimientos previos frente a un $8 \%$ de la universidad privada. Un $7 \%$ de las asignaturas de la universidad pública requiere la necesidad de haber cursado anteriormente alguna asignatura frente a un $8 \%$ de la universidad privada.

- Necesidad de conocimientos previos

- Necesidad de haber cursado una asignatura previa

- Ninguna recomendación

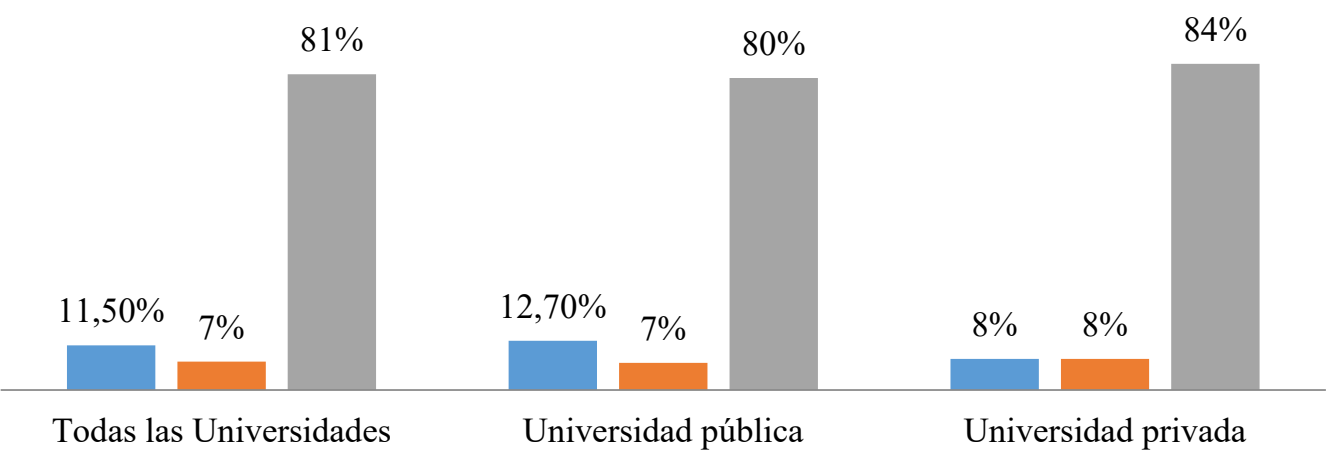

Figura 2. Recomendaciones.

Con relación al número de competencias específicas relacionadas con las diferentes asignaturas, como se puede apreciar en la Tabla 5 son muy dispares y están comprendidas entre 1 competencia específica y 15 competencias específicas. Además, 4 guías no especificaban el número de competencias específicas que estaban relacionadas con la materia. Cabe destacar que el $22.9 \%$ de las asignaturas tienen 6 competencias específicas. Se puede apreciar como en la universidad privada, hay un mayor porcentaje de competencias específicas en el rango 6-10, con un $72 \%$ respecto a la pública, que tiene un $43.6 \%$ en ese mismo rango. Por el contrario, la universidad pública tiene más competencias específicas en el rango 0-5 con un $45 \%$ respecto a la privada, con un $20 \%$ en ese mismo rango. 
Tabla 5

Número de competencias específicas relacionadas con la materia

\begin{tabular}{ccccccc}
\hline Rangos & \multicolumn{2}{c}{ Todas las universidades } & \multicolumn{2}{c}{ Universidad Pública } & \multicolumn{2}{c}{ Universidad Privada } \\
\hline & Frecuencia & Porcentaje & Frecuencia & Porcentaje & Frecuencia & Porcentaje \\
\hline $0-5$ & 39 & 40.7 & 32 & 45 & 7 & 20 \\
$6-10$ & 47 & 49 & 29 & 43.6 & 20 & 72 \\
$11-15$ & 10 & 14.3 & 8 & 11.4 & 2 & 8 \\
Total & 96 & 100.0 & 71 & 100.0 & 25 & 100.0 \\
\hline
\end{tabular}

En cuanto al número de resultados de aprendizaje u objetivos relacionados con la materia, como se puede comprobar en la Tabla 6 ocurre lo mismo que con las competencias específicas ya que es dispar y varía desde 2 objetivos o resultados de aprendizaje hasta 22. Cabe destacar dos aspectos, el primero es que el $18.8 \%$ de las guías analizadas no especifica los resultados de aprendizaje y objetivos relacionados con la materia. El segundo aspecto es que el $14.6 \%$ de las guías tienen 6 objetivos o resultados de aprendizaje, el valor más repetido. Los porcentajes en la universidad pública y privada son parecidos y siguen la misma tendencia. Cabe destacar que el $8 \%$ de las asignaturas de la universidad privada tienen entre 16 y 22 resultados de aprendizaje u objetivos relacionados con la materia, mientras que en la universidad pública no hay ninguno.

Tabla 6

Número de resultados de aprendizaje y objetivos relacionados con la materia

\begin{tabular}{ccccccc}
\hline Rangos & \multicolumn{2}{c}{ Todas las universidades } & \multicolumn{2}{c}{ Universidad Pública } & \multicolumn{2}{c}{ Universidad Privada } \\
\hline & Frecuencia & Porcentaje & Frecuencia & Porcentaje & Frecuencia & Porcentaje \\
\hline $0-5$ & 42 & 43.9 & 30 & 42.2 & 12 & 48 \\
$6-10$ & 34 & 35.5 & 26 & 36.5 & 8 & 32 \\
$11-15$ & 18 & 18.8 & 15 & 21.1 & 3 & 12 \\
$16-22$ & 2 & 2.0 & - & - & 2 & 8 \\
Total & 96 & 100.0 & 71 & 100.0 & 25 & 100.0 \\
\hline
\end{tabular}

\subsection{Uso de contenidos específicos musicales}

Con referencia a los contenidos, como se puede apreciar en la Figura 3 el 27.1\% de las guías incluyen Investigación en Educación musical como uno de sus contenidos, el $60.4 \%$ incluye audición, el 76\% incluye La música en el currículum de Educación Infantil, el 21.9\% Historia de la Música, el 56.3\% Interpretación Musical, el 65.6\% Expresión corporal (Danza y movimiento), el $50 \%$ Lenguaje Musical, el $68.8 \%$ Expresión vocal (Fisiología de la canto, canto, etc.), el $41.7 \%$ Parámetros del sonido, el 44.8\% Repertorio de Canciones, el 25\% Música y TIC, el 24\% Didáctica de las canciones, el $9.4 \%$ Creatividad e improvisación y el $11.5 \%$ otros contenidos. Los otros contenidos que no venían reflejados en el Protocolo pero sí en algunas Guías Didácticas eran Comunicación artística musical, Didáctica de la Música, El juego musical, Elaboración de Programaciones Didácticas, Fundamentos básicos de la expresión musical, Inteligencia musical, Música en los espectáculos, Musicoterapia y Normativa.

Una de las diferencias más significativas entre la universidad pública y privada es que en la universidad pública, el $78.9 \%$ de las asignaturas imparte como contenido la música en el currículo de Educación Infantil, por un $38 \%$ de la universidad privada. Además, la universidad pública, en el $28.2 \%$ de sus asignaturas imparte el contenido música y TIC, por un $12 \%$ de la universidad privada. En esta misma línea, la universidad pública imparte en el $66.2 \%$ de sus asignaturas el contenido de audición, por un $44 \%$ de la universidad privada. Por el contrario, en 
el 32\% de las asignaturas de la universidad privada se imparte el contenido de Historia de la Música, por un $18.3 \%$ de la universidad pública. El resto de contenidos analizados siguen patrones similares tanto en la universidad pública como en la universidad privada.

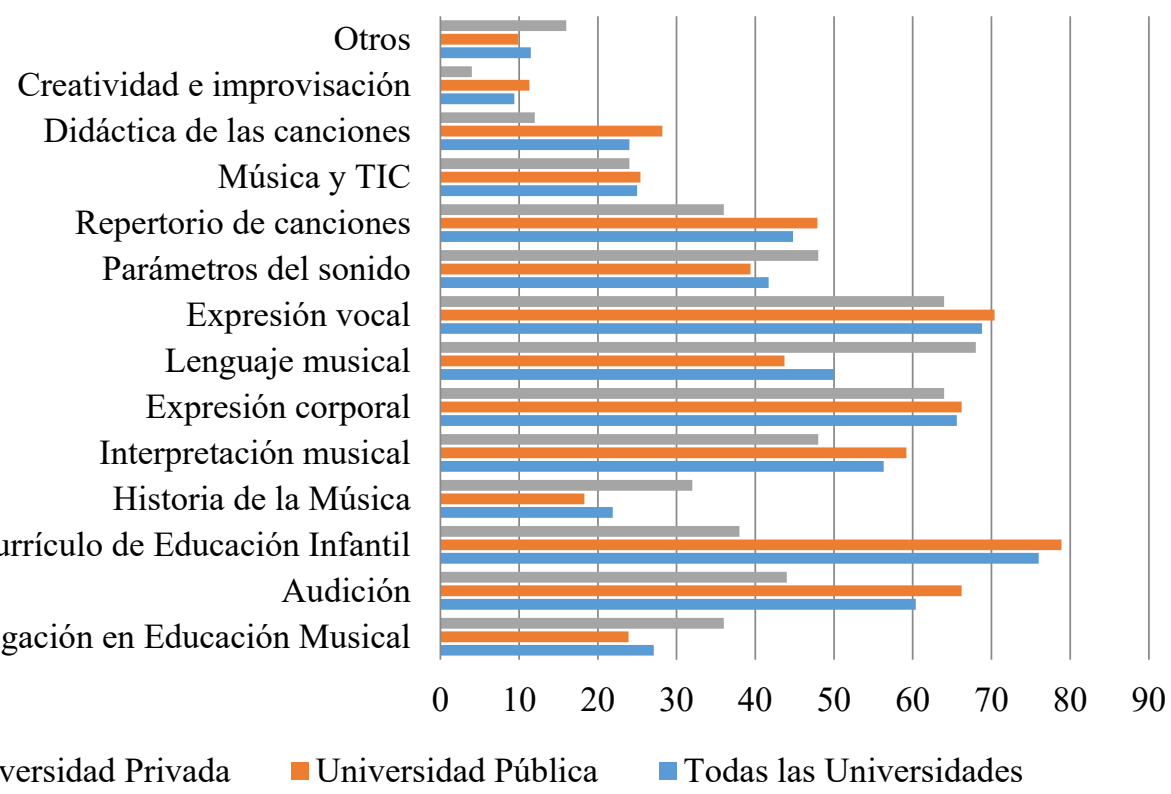

Figura 3. Contenidos específicos de las asignaturas.

\subsection{Uso de la metodología de enseñanza}

En cada asignatura se utilizan varias metodologías, el $95.8 \%$ utiliza la Exposición magistral, el 88.5\% las clases prácticas, el $72.9 \%$ los trabajos en grupo, el $57.3 \%$ los seminarios, el $45.8 \%$ las exposiciones, el $25 \%$ la discusión y el debate, el $25 \%$ la búsqueda y consulta de material bibliográfico, el 9.4\% el visionado de materiales audiovisuales, el 6.3\% la elaboración de ensayos, un $1 \%$ la clase invertida y un $4.2 \%$ otras que serían el estudio de casos, la resolución de problemas y el trabajo autónomo (Figura 4).

Se aprecia como todos los porcentajes de metodologías son superiores en la pública que en la privada, excepto la búsqueda y consulta de material bibliográfico, que en la universidad privada es de un $36 \%$ y en la universidad pública de un $21.1 \%$. También cabe destacar que la única asignatura en la que se utiliza la metodología de clase invertida pertenece a la universidad privada. Por el contrario, la universidad privada no utiliza dentro de sus metodologías la realización de ensayos, mientras que, en la pública, es del $8.5 \%$. El resto de las metodologías siguen tendencias parecidas en cuanto a sus porcentajes, aunque en todos es ligeramente superior la universidad pública. 
Exposición magistral

Discusión y debate

Visionado de maetriales audiovisuales

Exposiciones

Clases prácticas

Búsqueda y consulta de material bibliográfico

Elaboración de ensayos

Seminarios

Trabajos en grupo

Clase inversida

Otros
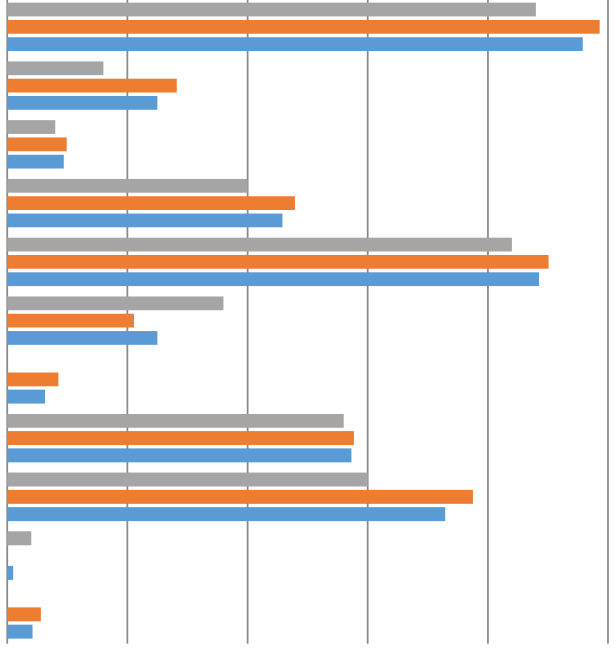

0

20

40

60

$80 \quad 100$

120

- Universidad Privada $\quad$ Universidad Pública $\quad$ Todas las universidades

Figura 4. Metodologías utilizadas.

\subsection{Uso de la evaluación}

En relación a los sistemas de evaluación utilizados, ocurre lo mismo que con la metodología, y cada asignatura tiene varias formas de evaluar. El 16.7\% evalúa la Audición (Tanto pruebas prácticas como actividades en clase), el 34.4\% evalúa la Interpretación (Tanto pruebas prácticas como actividades en clase), el $79.2 \%$ las Pruebas escritas, el $91.7 \%$ la Realización de actividades (Diseño de Unidades Didácticas, Elaboración de un blog, etc.), el $71.9 \%$ evalúa la asistencia y participación activa y el $8.3 \%$ evalúa otros elementos como la asistencia a visitas técnicas, exposiciones tanto individuales como grupales, uso de las TIC y autoevaluaciones (Tabla 7).

Una diferencia entre la universidad pública y privada es que, en la universidad pública, el $81.7 \%$ de las asignaturas tienen como sistema de evaluación la realización de una prueba escrita mientras que en la universidad privada es de un $72 \%$. Otro dato relevante es que en la universidad pública el $77.5 \%$ de las asignaturas usan como sistema de evaluación la asistencia y la participación activa, por un $56 \%$ de la universidad privada.

Tabla 7

Sistemas de evaluación

\begin{tabular}{|c|c|c|c|c|c|c|}
\hline \multirow[t]{2}{*}{ Sistema } & \multicolumn{2}{|c|}{ Todas las universidades } & \multicolumn{2}{|c|}{ Universidad Pública } & \multicolumn{2}{|c|}{ Universidad Privada } \\
\hline & Frecuencia & Porcentaje & Frecuencia & Porcentaje & Frecuencia & Porcentaje \\
\hline Audición & 16 & 16.7 & 14 & 19.7 & 2 & 8.0 \\
\hline Interpretación & 33 & 34.4 & 27 & 38.0 & 6 & 24.0 \\
\hline $\begin{array}{l}\text { Pruebas } \\
\text { escritas }\end{array}$ & 76 & 79.2 & 58 & 81.7 & 18 & 72.0 \\
\hline $\begin{array}{c}\text { Realización de } \\
\text { actividades }\end{array}$ & 88 & 91.7 & 66 & 93.0 & 22 & 88.0 \\
\hline $\begin{array}{c}\text { Asistencia y } \\
\text { participación } \\
\text { activa }\end{array}$ & 69 & 71.9 & 55 & 77.5 & 14 & 56.0 \\
\hline Otros & 8 & 8.3 & 5 & 7.0 & 3 & 12.0 \\
\hline
\end{tabular}


La ponderación que se atribuye a cada uno de los sistemas de evaluación, como se puede apreciar en la Tabla 8 varía mucho de unas guías a otras. La media de los porcentajes sería: $14.21 \%$ de la nota correspondería a audición, el 27,65\% a interpretación, el 38.64\% a la realización de pruebas escritas, el $47.02 \%$ a la realización de actividades, el $11.08 \%$ a la asistencia y participación activa, y el $13.0 \%$ a otros sistemas de evaluación.

Como podemos observar en las Tablas 9 y 10, las grandes diferencias en las ponderaciones de los sistemas de evaluación entre la universidad pública y la universidad privada es que la universidad pública, en un $43.5 \%$ de sus guías pondera la interpretación frente a un $24 \%$ de guías de la universidad privada, además, en la universidad pública el $25.3 \%$ de guías contempla en su ponderación la audición, frente a un $4 \%$ de la privada.

Se aprecia como la universidad pública tiene unas ponderaciones más altas en los sistemas de evaluación de pruebas escritas, asistencia y participación.

Tabla 8

Ponderación Sistemas de todas las universidades

\begin{tabular}{ccccccccccccc}
\hline & \multicolumn{2}{c}{ Audición } & \multicolumn{2}{c}{ Interpretación } & \multicolumn{2}{c}{$\begin{array}{c}\text { Pruebas } \\
\text { escritas }\end{array}$} & $\begin{array}{c}\text { Realización de } \\
\text { actividades }\end{array}$ & \multicolumn{2}{c}{$\begin{array}{c}\text { Asistencia y } \\
\text { participación }\end{array}$} & Otros \\
\hline Ponderación & F & P & F & P & F & P & F & P & F & P & F & P \\
\hline 0 & 7 & 7.3 & 2 & 2.1 & 3 & 3.1 & - & - & 8 & 8.3 & - & - \\
5 & - & - & - & - & 1 & 1.0 & - & - & 7 & 7.3 & 2 & 2.1 \\
10 & 2 & 2.1 & 1 & 1.0 & - & - & 3 & 3.1 & 19 & 19.8 & 1 & 1.0 \\
15 & 3 & 3.1 & 5 & 5.2 & 1 & 1.0 & - & - & 7 & 7.3 & - & - \\
20 & 3 & 3.1 & 6 & $6-3$ & 4 & 4.2 & 8 & 8.3 & 7 & 7.3 & 1 & 1.0 \\
25 & 1 & 2.1 & 6 & 6.3 & 6 & 6.3 & 7 & 7.3 & 1 & 1.0 & 1 & 1.0 \\
30 & 2 & 3.1 & 9 & 9.4 & 18 & 18.7 & 4 & 4.2 & 1 & 1.0 & - & - \\
35 & - & - & - & - & 1 & 1.0 & 2 & 2.1 & - & - & - & - \\
40 & - & - & 4 & 4.2 & 9 & 9.4 & 14 & 14.6 & 1 & 1.0 & - & - \\
45 & - & - & - & - & 1 & 1.0 & 5 & 5.2 & - & - & - & - \\
50 & - & - & 2 & 2.1 & 20 & 20.8 & 18 & 18.8 & - & - & - & - \\
55 & - & - & - & - & 1 & 1.0 & 1 & 1.0 & - & - & - & - \\
60 & 1 & 2.1 & - & - & 6 & 6.3 & 6 & 6.3 & - & - & - & - \\
65 & - & - & - & - & - & - & - & - & - & - & - & - \\
70 & - & - & 2 & 2.1 & 2 & 2.1 & 5 & 5.2 & - & - & - & - \\
75 & - & - & - & - & - & - & 2 & 2.1 & - & - & - & - \\
80 & - & - & - & - & - & - & 2 & 2.1 & - & - & - & - \\
85 & - & - & - & - & - & - & - & - & - & - & - & - \\
90 & - & - & - & - & 1 & 1.0 & 1 & 1.0 & - & - & - & - \\
95 & - & - & - & - & - & - & 1 & 1.0 & - & - & - & - \\
100 & - & - & - & - & - & - & 4 & 4.2 & - & - & - & - \\
\hline
\end{tabular}

Nota: $F=$ frecuencia y $P=$ porcentaje.

Tabla 9

Ponderación Sistemas Universidad Pública

\begin{tabular}{ccccccccccccc}
\hline & Audición & Interpretación & \multicolumn{2}{c}{$\begin{array}{c}\text { Pruebas } \\
\text { escritas }\end{array}$} & \multicolumn{2}{c}{$\begin{array}{c}\text { Realización de } \\
\text { actividades }\end{array}$} & \multicolumn{2}{c}{$\begin{array}{c}\text { Asistencia y } \\
\text { participación }\end{array}$} & Otros \\
\hline Ponderación & $\mathrm{F}$ & $\mathrm{P}$ & $\mathrm{F}$ & $\mathrm{P}$ & $\mathrm{F}$ & $\mathrm{P}$ & $\mathrm{F}$ & $\mathrm{P}$ & $\mathrm{F}$ & $\mathrm{P}$ & $\mathrm{F}$ & $\mathrm{P}$ \\
\hline 0 & 7 & 9.9 & 2 & 2.8 & 3 & 4.2 & - & - & 8 & 11.3 & - & - \\
5 & - & - & - & - & 1 & 1.4 & - & - & 6 & 8.5 & 1 & 1.4 \\
10 & 2 & 2.8 & 1 & 1.4 & - & - & 2 & 2.8 & 12 & 16.9 & - & -
\end{tabular}




\begin{tabular}{ccccccccccccc}
\hline & \multicolumn{2}{c}{ Audición } & \multicolumn{2}{c}{ Interpretación } & \multicolumn{2}{c}{$\begin{array}{c}\text { Pruebas } \\
\text { escritas }\end{array}$} & $\begin{array}{c}\text { Realización de } \\
\text { actividades }\end{array}$ & \multicolumn{2}{c}{$\begin{array}{c}\text { Asistencia y } \\
\text { participación }\end{array}$} & Otros \\
\hline Ponderación & F & P & F & P & F & P & F & P & F & P & F & P \\
\hline 15 & 3 & 4.2 & 5 & 7.0 & 1 & 1.4 & - & - & 6 & 8.5 & - & - \\
20 & 2 & 2.8 & 4 & 5.6 & 4 & 5.6 & 6 & 8.5 & 5 & 7.0 & 1 & 1.4 \\
25 & 1 & 1.4 & 5 & 7.0 & 6 & 8.5 & 6 & 8.5 & 1 & 1.4 & 1 & 1.4 \\
30 & 2 & 2.8 & 8 & 11.3 & 14 & 19.7 & 3 & 4.2 & 1 & 1.4 & - & - \\
35 & - & - & - & - & 1 & 1.4 & 1 & 1.4 & - & - & - & - \\
40 & - & - & 4 & 5.6 & 5 & 7.0 & 10 & 14.1 & 1 & 1.4 & - & - \\
45 & - & - & - & - & 1 & 1.4 & 4 & 5.6 & - & - & - & - \\
50 & - & - & 1 & 1.4 & 16 & 22.5 & 14 & 19.7 & - & - & - & - \\
55 & - & - & - & - & - & - & 1 & 1.4 & - & - & - & - \\
60 & 1 & 1.4 & - & - & 3 & 4.2 & 3 & 4.2 & - & - & - & - \\
65 & - & - & - & - & - & - & - & - & - & - & - & - \\
70 & - & - & 1 & 1.4 & - & - & 4 & 5.6 & - & - & - & - \\
75 & - & - & - & - & - & - & 2 & 2.8 & - & - & - & - \\
80 & - & - & - & - & - & - & 1 & 1.4 & - & - & - & - \\
85 & - & - & - & - & - & - & - & - & - & - & - & - \\
90 & - & - & - & - & 1 & 1.4 & - & - & - & - & - & - \\
95 & - & - & - & - & - & - & 1 & 1.4 & - & - & - & - \\
100 & - & - & - & - & - & - & 3 & 4.2 & - & - & - & - \\
\hline
\end{tabular}

Nota: F=frecuencia y $\mathrm{P}=$ porcentaje.

Tabla 10

Ponderación Sistemas Universidad Privada

\begin{tabular}{|c|c|c|c|c|c|c|c|c|c|c|c|c|}
\hline \multirow[b]{2}{*}{ Ponderación } & \multicolumn{2}{|c|}{ Audición } & \multicolumn{2}{|c|}{ Interpretación } & \multicolumn{2}{|c|}{$\begin{array}{l}\text { Pruebas } \\
\text { escritas }\end{array}$} & \multicolumn{2}{|c|}{$\begin{array}{c}\text { Realización de } \\
\text { actividades }\end{array}$} & \multicolumn{2}{|c|}{$\begin{array}{l}\text { Asistencia y } \\
\text { participación }\end{array}$} & \multicolumn{2}{|c|}{ Otros } \\
\hline & $\mathrm{F}$ & $P$ & $\mathrm{~F}$ & $P$ & $\mathrm{~F}$ & $P$ & $\mathrm{~F}$ & $P$ & $\mathrm{~F}$ & $P$ & $\mathrm{~F}$ & $P$ \\
\hline 0 & - & - & - & - & - & - & - & - & - & - & - & - \\
\hline 5 & - & - & - & - & - & - & - & - & 1 & 4.0 & 1 & 4.0 \\
\hline 10 & - & - & - & - & - & - & 1 & 4.0 & 7 & 28.0 & - & - \\
\hline 15 & - & - & - & - & - & - & - & - & 1 & 4.0 & - & - \\
\hline 20 & 1 & 4.0 & 2 & 8.0 & - & - & 2 & 8.0 & 2 & 8.0 & - & - \\
\hline 25 & - & - & 1 & 4.0 & - & - & 1 & 4.0 & - & - & - & - \\
\hline 30 & - & - & 1 & 4.0 & 4 & 16.0 & 1 & 4.0 & - & - & - & - \\
\hline 35 & - & - & - & - & - & - & 1 & 4.0 & - & - & - & - \\
\hline 40 & - & - & - & - & 4 & 16.0 & 4 & 16.0 & - & - & - & - \\
\hline 45 & - & - & - & - & - & - & 1 & 4.0 & - & - & - & - \\
\hline 50 & - & - & 1 & 4.0 & 4 & 16.0 & 4 & 16.0 & - & - & - & - \\
\hline 55 & - & - & - & - & 1 & 4.0 & - & - & - & - & - & - \\
\hline 60 & - & - & - & - & 3 & 12.0 & 3 & 12.0 & - & - & - & - \\
\hline 65 & - & - & - & - & - & - & - & - & - & - & - & - \\
\hline 70 & - & - & 1 & 4.0 & 2 & 8.0 & 1 & 4.0 & - & - & - & - \\
\hline 75 & - & - & - & - & - & - & - & - & - & - & - & - \\
\hline 80 & - & - & - & - & - & - & 1 & 4.0 & - & - & - & - \\
\hline 85 & - & - & - & - & - & - & - & - & - & - & - & - \\
\hline 90 & - & - & - & - & - & - & 1 & 4.0 & - & - & - & - \\
\hline 95 & - & - & - & - & - & - & - & - & - & - & - & - \\
\hline 100 & - & - & - & - & - & - & 1 & 4.0 & - & - & - & - \\
\hline
\end{tabular}

Nota: F=frecuencia y $\mathrm{P}=$ porcentaje. 


\subsection{Uso de la bibliografía}

En cuanto al tipo de referencias bibliográficas podemos apreciar en la Tabla 11 como el 87.5\% de las guías tienen bibliografía básica, el 51\% tienen bibliografía específica y el $51 \%$ tienen ambas bibliografías. Por lo que un $\mathbf{1 2 . 5 \%}$ de las guías no tienen ningún tipo de bibliografía.

En casi el total de las universidades privadas, las guías docentes tienen bibliografía básica, más concretamente el $96 \%$ frente a un $84.5 \%$ de la universidad pública. También en la bibliografía específica el porcentaje de guías docentes que tienen bibliografía específica es mayor en la privada que en la pública, un $60 \%$ frente a un $47.9 \%$ respectivamente.

Tabla 11

Tipos de Bibliografía

\begin{tabular}{ccccccc}
\hline Tipos & \multicolumn{2}{c}{ Todas las universidades } & \multicolumn{2}{c}{ Universidad Pública } & \multicolumn{2}{c}{ Universidad Privada } \\
\hline & Frecuencia & Porcentaje & Frecuencia & Porcentaje & Frecuencia & Porcentaje \\
\hline Básica & 84 & 87.5 & 60 & 84.5 & 24 & 96.0 \\
Específica & 49 & 51.0 & 34 & 47.9 & 15 & 60.0 \\
Ambas & 49 & 51.0 & 34 & 47.9 & 15 & 60.0 \\
\hline
\end{tabular}

Como podemos apreciar en la Tabla 12, la cantidad de referencias bibliográficas es muy dispar, y varía entre 2 y 50. La media de referencias bibliográficas sería de 18. No se aprecian diferencias significativas entre la universidad pública y la privada. Se puede destacar que la universidad pública tiene mayor número de referencias bibliográficas en el rango 11-20, mientras que la universidad privada tiene mayor número de referencias bibliográficas en el rango 21-30.

Tabla 12

Cantidad de Referencias Bibliográficas

\begin{tabular}{ccccccc}
\hline Rangos & \multicolumn{2}{c}{ Todas las universidades } & \multicolumn{2}{c}{ Universidad Pública } & \multicolumn{2}{c}{ Universidad Privada } \\
\hline & Frecuencia & Porcentaje & Frecuencia & Porcentaje & Frecuencia & Porcentaje \\
\hline $0-10$ & 31 & 32.2 & 23 & 32.4 & 8 & 32.0 \\
$11-20$ & 33 & 34.4 & 26 & 36.6 & 7 & 28.0 \\
$21-30$ & 16 & 16.7 & 10 & 14.1 & 6 & 24.0 \\
$31-50$ & 16 & 16.7 & 12 & 16.9 & 4 & 16.0 \\
\hline
\end{tabular}

\section{Discusión}

Tras los resultados obtenidos se aprecia una diferencia significativa en la elaboración de las Guías Docentes de los Grados de Educación Infantil de las universidades públicas y privadas de España. Tanto en los aspectos generales como en los distintos aspectos curriculares.

El grado de similitud a la hora de dar importancia a la música en el Grado de Educación Infantil es bastante alto ya que la mayoría de universidades, dedican varias asignaturas a la Educación Musical. Esto corrobora las palabras de Frega (citada en Arguedas, 2004), que argumenta la necesidad de realizar en las aulas de Educación Infantil actividades auditivas, expresivas, rítmicas, de creación e interpretación, para conseguir los objetivos musicales que se pretenden alcanzar. Si los maestros de Educación Infantil no están formados en Educación musical no pueden aplicarla en sus aulas.

Se reafirma la importancia otorgada a la Educación Musical en el Grado de Educación Infantil al constatar que el $66.7 \%$ de las asignaturas son de carácter obligatorio. Este dato es aún mayor 
en la universidad privada donde alcanza el $84 \%$. En cambio, queda contrarrestado al comprobar que el $93.8 \%$ de las asignaturas se imparten de manera cuatrimestral/semestral. En el caso de la universidad privada, el porcentaje es aún mayor y alcanza el $100 \%$, por lo que no se cursa ninguna asignatura de Educación Musical de forma anual.

En cuanto a los contenidos que se trabajan dentro de las asignaturas, nuestro estudio constata las investigaciones previas realizadas por Vicente y Rodríguez (citados en García-Gil y Bernabé, 2019) que señalaban la necesidad de promover en beneficio del alumnado de Infantil, la investigación-acción para potenciar a su vez, la creación de materiales específicos, el intercambio y el estudio sobre la práctica.

Nuestro estudio sigue la línea de las investigaciones realizadas por López (2008) y Carbajo (2009) que coinciden en recomendar la interpretación y el trabajo sobre la voz como contenidos musicales imprescindibles en el aula de Educación Infantil.

Podemos apreciar en nuestro estudio la poca importancia que las asignaturas de educación musical dan a la creatividad. Esto contradice la idea de Madsen (2000) cuando afirma que la música es fruto de la creatividad del ser humano mostrándose como producto cultural, artístico y técnico al mismo tiempo. Esta afirmación es apoyada por Alsina y De la Creu (2009) y Carrillo (2015) que han reflexionado sobre el desarrollo de la competencia creativa y que han llegado a la conclusión de su relevancia para el maestro de Educación Infantil. Además, estos autores añadieron la interpretación, la dirección instrumental y el análisis musical. En esta dirección, Rodríguez (2015) realizó un estudio que tuvo como conclusión la necesidad de formar a los docentes en la competencia creativa, ya que le va a repercutir tanto en su formación, como en su práctica.

La mayoría de las guías docentes no realizan una distinción entre objetivos y resultados de aprendizaje siguiendo la línea de las investigaciones realizadas por Nava, Pazos, de la Cruz y Sánchez-Oro (2014). Estos autores afirman que en la mayoría de las ocasiones estos dos conceptos son utilizados de forma indistinta como sinónimos, debido a que en la mayoría de ocasiones los objetivos son escritos en función del aprendizaje previsto.

Se puede observar en nuestro estudio como la metodología utilizada, en un $95.8 \%$ de las asignaturas es la exposición magistral, por lo que se confirma el estudio realizado por Vallejo y Molina (2011) a 118 alumnos del Grado de Educación Infantil de la Universidad de Murcia sobre las metodologías que se impartían en dicho grado. Los resultados arrojaron que un $66.95 \%$ de las asignaturas utilizaban la exposición magistral a la hora de impartir sus clases. Esto contradice las palabras de Molina e Illán (citados en Vallejo y Molina, 2011) al afirmar que el nuevo modelo educativo, debe enfocar su proceso de enseñanza-aprendizaje en un trabajo de cooperación entre docentes y alumnos, que esté orientado al aprendizaje autónomo del alumno. Podemos comprobar cómo 25 años después se siguen utilizando las mismas metodologías que se utilizaban en el estudio realizado por Prégent (1995) y que no se ha avanzado mucho hacia las metodologías activas que basan el aprendizaje en el alumno.

Con relación a la evaluación, se observa cómo el $79.2 \%$ de las asignaturas siguen como modelo de evaluación la realización de una prueba escrita y que, además, tiene una gran ponderación en la nota final por lo que, no está en la línea de la investigación realizada por Álvarez, Grau y Tortosa (2010) que apuntan que el desarrollo de sistemas de evaluación formativa en la universidad mejora la implicación del alumnado. Son numerosos los estudios en contra de nuestros datos. Por ejemplo, para Montero, Villalobos y Valverde (2007), existen mejores resultados académicos cuando los alumnos se implican en la evaluación.

Un estudio realizado por Martínez y Flores (2014) obtuvo unos resultados similares a los obtenidos en nuestra investigación. En su estudio, según la opinión del alumnado del Grado de Educación infantil, los instrumentos de evaluación más utilizados por el profesorado para 
realizar la evaluación serían los exámenes escritos, trabajos en grupo, carpetas de aprendizaje o portafolio y diarios. En dicho estudio, los profesores reconocían que el instrumento más utilizado para la evaluación del alumnado es el examen.

Podemos apreciar que la diferencia más significativa entre la universidad pública y la privada es la opción relacionada con el idioma. La posibilidad de estudiar de forma bilingüe solo se oferta en la universidad privada. En este sentido, nuestro estudio constata las investigaciones de Algaba (2015), cuando afirma que las ventajas asociadas a la universidad privada se refieren, entre otras cosas, a una oferta bilingüe más amplia.

Se ha podido observar si existe relación y similitud entre lo que aprenden los alumnos del Grado de Educación Infantil y lo que posteriormente tienen que enseñar a sus alumnos como fututos maestros. En este aspecto, el Real Decreto 1630/2006 de 29 de diciembre, por el que se establecen las enseñanzas mínimas del segundo ciclo de Educación Infantil no es lo suficientemente específico en cuanto a los contenidos que se deben desarrollar en educación musical, pero podemos destacar cuatro contenidos en el Bloque 3 del citado Real que presentan una relación entre los contenidos específicos musicales presentes en las guías docentes y en la normativa aplicable en el aula. Se aprecia un grado de relación, ya que se obtuvo que el $68.8 \%$ de las asignaturas de Educación Musical imparten expresión vocal. Ocurre lo mismo con la interpretación musical y la expresión corporal, los resultados revelan que un 59.2\% y un $66.2 \%$ respectivamente de las asignaturas imparten este tipo de contenidos. Ocurre lo mismo con contenidos como la Audición y el Reconocimiento de sonidos. Los resultados muestran que, el $66.2 \%$ de las asignaturas del Grado de Educación Infantil trabajan la audición como uno de sus contenidos y $41.7 \%$ de las asignaturas contempla contenidos relacionados con el Reconocimiento sonoro.

\section{Conclusiones}

Como conclusiones al objetivo general destacar que vistos los resultados obtenidos por el Protocolo de Análisis de Guías Docentes, se puede observar que, a la hora de elaborar las Guías Docentes, no existe un criterio unánime para ello, aunque la mayoría contienen los mismos apartados son muy dispares en su contenido.

Respecto a las conclusiones obtenidas con relación al objetivo específico 1, hemos comprobado como la oferta de asignaturas de la universidad pública es mucho mayor que la de la privada, pero en ambas no se sigue un indicador común para dar nombre a la asignatura, por lo que encontramos una gran diversidad y variedad de nombres distintos. Pero si se puede apreciar como la mayoría llevan incluido el término "música" o "musical". En cuanto al curso donde se imparten las asignaturas, se ha visto que el curso con mayor presencia de asignaturas es 3 , seguido de 4으, por lo que se aprecia la frecuencia de incluir las asignaturas de Educación musical en los años más avanzados del Grado de Educación Infantil. La mayor parte de las asignaturas tienen un carácter obligatorio y su temporalidad es semestral en su mayoría, aunque se reparten de manera equitativa entre el primer y el segundo semestre. Por su carácter obligatorio, vemos la importancia que posee la música en la formación del maestro. Esto queda contradicho con la temporalidad de las asignaturas. Al ser obligatorias, deberían tener un mayor peso en el Grado de Educación Infantil y por tanto ampliar sus contenidos y el número de créditos ECTS para ser anual. Otro aspecto a destacar es la asiduidad de carga lectiva que tienen las asignaturas, siendo predominante la carga lectiva de 6 Créditos ECTS, aunque encontramos asignaturas que varían desde los 3 hasta los 1 créditos. Los últimos aspectos generales para resaltar serían el idioma en que se imparten, que en un gran porcentaje es el español, y que no requieren de ninguna recomendación previa para poder cursar la asignatura. 
Con relación al segundo objetivo específico, hemos observado cómo existe una gran disparidad en cuanto al número de competencias específicas relacionadas con la materia que se encuentran en las Guías Docentes, ya que varían desde 0 hasta 15. Por lo que no existe un patrón común a la hora de establecerlas. Nuestro análisis se realizó por rangos, y el más frecuente es el rango de competencias específicas 6-10. Durante el estudio del Protocolo de Análisis de Guías Docentes, se apreció como las guías no diferencian los resultados específicos de aprendizaje con los objetivos, por lo que se analizó conjuntamente. Al igual que con las competencias específicas, los resultados de aprendizaje/objetivos no siguen ningún patrón y son muy dispares en número entre unas asignaturas y otras ya que varían desde 0 hasta 22. El análisis se llevó a cabo por rangos y el más repetido fue el rango de resultados de aprendizaje/objetivos 0-5. La mayor parte de las asignaturas tienen unos contenidos parecidos, y es el aspecto más igualitario en el conjunto de Guías Docentes. Los contenidos más frecuentes son la audición, expresión vocal, la música en el currículo de Educación Infantil y la expresión corporal. Por el contrario, los menos utilizados son la creatividad e improvisación, la investigación en Educación Musical y la historia de la música. Desde el punto de la metodología para abordar las clases, en el estudio se ha llegado a la conclusión que no ha evolucionado la forma de impartir las clases ya que, aunque se imparten clases prácticas, la mayoría siguen siendo exposiciones magistrales y no se imparten clases con nuevas metodologías que centren el proceso de enseñanza-aprendizaje en el alumno de una forma más activa. La forma de evaluar sigue el mismo patrón que la metodología y se sigue centrando en la realización de pruebas escritas y la realización de actividades. También se da importancia a la asistencia a clase y la participación activa, sobre todo en la universidad pública. En cuanto a la bibliografía que contienen las Guías Docentes, se ha llegado a observado como la mayoría poseen bibliografía básica, mientras que solo la mitad de ellas llevan incorporada una bibliografía específica. La cantidad de referencias es muy dispar entre unas guías y otras.

Como conclusiones al tercer objetivo específico destacamos que, se puede apreciar cómo la diferencia más significativa entre la universidad pública y la privada es la opción relacionada con el idioma. La posibilidad de estudiar de forma bilingüe, solo se oferta en la universidad privada. También se puede destacar que, en la universidad privada, todas las asignaturas de Educación Musical tienen una temporalidad semestral y que ninguna tiene carácter anual. Otra diferencia se aprecia en la importancia que la universidad pública da los contenidos musicales en el currículo de Educación Infantil mientras que, en la privada, más de la mitad de las asignaturas no los contemplan.

\section{Referencias bibliográficas}

Alaminos, A. (2006). El muestreo en la investigación social. En A. Alaminos, y J. L. Castejón (Comps.). Elaboración, análisis e interpretación de encuestas, cuestionarios y escalas de opinión (pp. 41-55). Alicante: Marfil.

Algaba, E. (2015). Universidad pública y privada en España: dos modelos distintos con objetivos similares. Encuentros Multidisciplinares, 49, 1-10,

Alsina, M., y De la Creu, J. (2009). Competencias creativas en el aula de música. Eufonía, 45,98107.

Álvarez, J.D., Grau, S. y Tortosa, M.T. (2010). Estrategias de coordinación metodológicas en la evaluación formativa de una asignatura. En C. Gómez \& S. Grau (Eds.). Evaluación de aprendizajes en el EEES, (pp.75-90). Alcoy: Marfil.

Arguedas, C. (2004). La expresión musical y el currículo escolar. Educación, 28(1), 111-122. 
Bojórquez, J.A., López, L., Hernández, M. E. y Jiménez, E. (Agosto de 2013). Utilización del alfa de Cronbach para validar la confiabilidad de un instrumento de medición de satisfacción del estudiante en el uso del software Minitab. Eleventh Latin American and Caribbean Conferencefor Engineering and Technology. Conferencia llevada a cabo en Cancún, México, 1-9.

Capistrán, R. W. (2016). La Educación Musical a Nivel Preescolar: El Caso del Instituto de Educación del Estado de Aguascalientes, México. Revista Internacional de Educación Musical, (4), 3-12.

Carbajo, C. (2009). El perfil profesional del docente de música de educación primaria: autopercepción de competencias profesionales y la práctica de aula. Murcia: Servicio de Publicaciones de la Universidad de Murcia

Carrillo, C. (2015). Competencias profesionales del profesorado de música: de los referentes teóricos a la concreción de una propuesta. Revista Internacional de Educación Musical, 3,11-21.

Celina, H. y Campo-Arias, A. (2005). Aproximación al uso del coeficiente alfade Cronbach. Revista Colombiana de Psiquiatría, 4, 572-580.

García-Gil, D. y Bernabé, M. M. (2019). Formación y preparación musical en el Grado de Educación Infantil: Consideraciones de las alumnas en Educación Superior. Revista Prisma, 25, 126-148.

García, M. et al (2001). Proyecto integral de calidad en las escuelas infantiles de titularidad municipal. Madrid: UCM -Ayuntamiento de Madrid.

Lizaso, M. B. (2017). Fundamentos psicopedagógicos y principios de intervención en Educación Infantil. En Cremades, R. (Coord.). Desarrollo de la expresión musical en Educación Infantil (pp. 1-20). Madrid: Paraninfo.

López, Ma A. (2008). La formación del maestro de educación infantil en la música y su enseñanza y su adecuación al espacio europeo de educación superior. Innovación Educativa, 18, 223237.

Madsen, C. K. (2000). Vision 2020: The House Wright symposium on the future of music education. Reston: MENC.

Martínez-Mínguez, L. y Flores, G. (2014). Profesorado y egresados ante los sistemas de evaluación del alumnado en la formación inicial del maestro de educación infantil. Revista Digital de Investigación en Docencia Universitaria, 8, 29-50.

McMillan, J. y Schumacher, S. (2005). Investigación educativa (5.a edición). Madrid: PEARSON EDUCACIÓN, S. A.

Montero, E., Villalobos, J. y Valverde, A. (2007). Factores institucionales, pedagógicos, psicosociales y sociodemográficos asociados al rendimiento académico en la Universidad de Costa Rica: Un análisis multinivel. RELIEVE, 13(2) ,215-234.

Navas, T., Pazos, A., de la Cruz, A. y Sánchez-Oro, J. J. (2014). Manual para la renovación de las guías docentes. Universidad complutense de Madrid. Recuperado de: https://eprints.ucm.es/27268/1/Manual\%20para\%20la\%20Renovacio\%CC\%81n\%20de\% 20las\%20Gui\%CC\%81as\%20Docentes\%20de\%20la\%20Facultad\%20de\%20Geografi\%CC\% 81a\%20e\%20Historia.pdf

Pastor, Francesc. (2009). Investigar en diseño curricular: estudio de caso de las guías docentes de la universidad de Alicante. (Tesis Doctoral). Universidad de Alicante: Alicante. 
Prégent, R. (1990). La preparation d'uncours. Montréal: Ediciones del'Ecole Polytechnique de Montréal.

Real Decreto 1630/2006, de 29 de diciembre, por el que se establecen las enseñanzas mínimas del segundo ciclo de Educación infantil.

Orden $\mathrm{ECl} / 3854 / 2007$, de 27 de diciembre, por la que se establecen los requisitos para la verificación de los títulos universitarios oficiales que habiliten para el ejercicio de la profesión de Maestro en Educación Infantil.

Rodríguez Lorenzo, G. A. (2015). Música, creación e interpretación: del aula universitaria al aula de educación infantil. Opción, 6, 742-764.

Sánchez-Báscones, M.; Ruiz-Esteban, C.; Pascual-Gómez, I. (2011). La guía docente como eje del proceso de enseñanza-aprendizaje. Bordón 63 (2), 53-64.

Vallejo, M. y Molina, J. (2011). Análisis de las metodologías activas en el grado de maestro en educación infantil: la perspectiva del alumnado. REIFOP, 14 (1) ,207-217.

Vejo, Primo. (2012). Herramientas para la evaluación, a través de indicadores, de las guías docentes universitarias: aplicación a los estudios de máster de ingeniería ambiental.

Vicente, R. Ma , y Rodríguez, J. (2014). Opinión y valoración del profesorado sobre los materiales didácticos de música en Educación Infantil. Bordón, 66(3), 149-163.

Wächter, B. (2004). The Bologna Process: developments and prospects. European Journal of Education, 39(3), 266-273.

Zabalza, M.A. (2011). Metodología docente. Revista de Docencia Universitaria. REDU. Monográfico: El espacio europeo de educación superior. ¿Hacia dónde va la Universidad Europea? 9(3), 75-98. Recuperado dehttp://redaberta.usc.es/redu 